\title{
Notes for the Reader
}

All Japanese and Chinese names are in standard order of family name first, followed by the given name.

Transliterations follow standard rules for each language; macrons and diacritics are not used for words and names commonly used in English (e.g., Kyoto, Tokyo, Shinto, mandala).

As a general rule, premodern Japanese dates (prior to 1873, when the Western calendar was introduced) are given in both traditional era names (nengō) and their rendering in the corresponding year of the Common Era (CE), followed, when relevant, by the lunisolar month and day. Thus, Bunmei 13 (1482) 2/18 refers to the thirteenth year of the Bunmei era (corresponding to $1482 \mathrm{CE}$ ), second month, eighteenth day. Note that month indications prior to January 1,1873 , do not correspond to any specific month in the Western calendar (i.e., the second month of the thirteenth year of the Bumei era is not equivalent to February 1482). 
